\title{
A Čech function in ZFC
}

by

Fred Galvin (Lawrence, KS) and Petr Simon (Praha)

\begin{abstract}
A nontrivial surjective Čech closure function is constructed in ZFC.
\end{abstract}
A closure function on a set $X$ is a mapping $\varphi: \mathcal{P}(X) \rightarrow \mathcal{P}(X)$ such that (i) $\varphi(\emptyset)=\emptyset$, (ii) $P \subseteq \varphi(P)$ for all $P \subseteq X$, and (iii) $\varphi(P \cup Q)=\varphi(P) \cup \varphi(Q)$ for all $P, Q \subseteq X$. In 1947 Eduard Čech [Č] asked: is there a closure function, other than the identity, which is onto $\mathcal{P}(X)$ ? A mapping answering Cech's question has come to be called a Čech function.

In 1978 the first author [G] announced a solution; however, dissatisfied that his solution required some set-theoretic assumptions, he delayed writing a paper. (Eventually, E. C. Milner and Karel Prikry [MP] included this construction of a Čech function in a survey paper on almost disjoint sets.) Around the same time, Roderick A. Price $[\mathrm{P}]$ produced a Cech function, assuming the continuum hypothesis, and remarked that the equality $\mathfrak{r}=\mathfrak{c}$ suffices. (Here $\mathfrak{r}$ is the reaping number, the smallest size of a family $\mathcal{R} \subseteq[\omega]^{\omega}$ such that for every $X \subseteq \omega$ there is some $R \in \mathcal{R}$ with either $R \subseteq X$ or $R \subseteq \omega \backslash X$.) However, as the inequality $\mathfrak{r}<\mathfrak{c}$ is consistent with ZFC ([K, VIII, Exercise A10]), Price's approach can hardly be modified into a ZFC version.

In 1981 the second author with B. Balcar and J. Dočkálková [BDS] constructed (in ZFC) a family of subsets of $\omega$ with the properties needed for the first author's construction of a Čech function, namely, an infinite completely separable almost disjoint family. Now, the first author's construction ostensibly used a stronger assumption, namely, the existence of an infinite completely separable maximal almost disjoint family; maybe this is why the two results were not connected for 25 years. We still do not know if this stronger statement is provable in ZFC. However, the second author recently observed that the first author's construction does not really need

2000 Mathematics Subject Classification: 54A05, 54G20, 04A20.

Key words and phrases: closure space, almost disjoint family. 
maximality, and so the existence of a Čech function on $\omega$ is proved in ZFC. A self-contained proof is given in this note.

1. Short version. We shall work on the set $\omega$ of all finite ordinals. An almost disjoint family is a family of infinite subsets of $\omega$ such that any two distinct members meet in a finite set. If $\mathcal{A}$ is an almost disjoint family, then it naturally divides all subsets of $\omega$ into two classes: a set is big if it has infinite intersection with infinitely many members of $\mathcal{A}$, and small otherwise. In symbols, the family of big sets is

$$
\mathcal{J}^{+}(\mathcal{A})=\{X \subseteq \omega:|\{A \in \mathcal{A}:|X \cap A|=\omega\}| \geq \omega\} .
$$

Clearly, the small sets, i.e. $\mathcal{J}(\mathcal{A})=\{X \subseteq \omega:|\{A \in \mathcal{A}:|X \cap A|=\omega\}|<\omega\}$, form an ideal in $\mathcal{P}(\omega)$. An almost disjoint family $\mathcal{A}$ on $\omega$ is completely separable if, for each $X \in \mathcal{J}^{+}(\mathcal{A})$, there is some $A \in \mathcal{A}$ with $A \subseteq X$. (It should be noted that the term "completely separable" has sometimes been used ([G], $[\mathrm{MP}])$ to mean, in effect, "completely separable and maximal". This is due to the fact that the original definition of a completely separable almost disjoint family given in $[\mathrm{H}]-\mathcal{A}$ is completely separable if for every $M \subseteq \omega$ either $M \subseteq \bigcup \mathcal{B}$ for some finite $\mathcal{B} \subseteq \mathcal{A}$ or there is some $A \in \mathcal{A}$ with $A \subseteq M$-implies maximality.)

Theorem 1 ([BDS, Corollary 2.3]). There is an infinite completely separable almost disjoint family on $\omega$.

OBSERVATION 1. If $\mathcal{A}$ is a completely separable almost disjoint family on $\omega$ and $X \in \mathcal{J}^{+}(\mathcal{A})$, then $|\{A \in \mathcal{A}: A \subseteq X\}|=\mathfrak{c}$.

Proof. Choose distinct sets $A_{n} \in \mathcal{A}, n \in \omega$, with $\left|X \cap A_{n}\right|=\omega$. For each $n \in \omega$ choose an infinite set $M_{n} \subseteq X \cap A_{n} \backslash \bigcup_{i<n} A_{i}$ with $\left|A_{n} \backslash M_{n}\right|=\omega$. Choose an almost disjoint family $\mathcal{B}$ of size $\mathfrak{c}$ on $\omega$. For each $B \in \mathcal{B}$, the set $\bigcup\left\{M_{n}: n \in B\right\}$ belongs to $\mathcal{J}^{+}(\mathcal{A})$ and, by the complete separability of $\mathcal{A}$, it contains some $A(B) \in \mathcal{A}$. Clearly $A(B) \neq A\left(B^{\prime}\right)$ for distinct $B, B^{\prime} \in \mathcal{B}$, and each $A(B)$ is a subset of $X$.

TheOrem 2 ([MP, Theorem 4.1]). If there is an infinite almost disjoint family $\mathcal{A}$ on $\omega$ such that for every $X \in \mathcal{J}^{+}(\mathcal{A})$ one has $|\{A \in \mathcal{A}: A \subseteq X\}|$ $=\mathfrak{c}$, then there is a Čech function $\varphi: \mathcal{P}(\omega) \rightarrow \mathcal{P}(\omega)$.

REMARK. Our Theorem 2 differs from Theorem 4.1 of [MP], in that the almost disjoint family is not assumed to be maximal, but it has essentially the same proof.

Corollary. There is a Čech function $\varphi: \mathcal{P}(\omega) \rightarrow \mathcal{P}(\omega)$.

Proof. Apply Theorem 1, Observation 1, and Theorem 2. 
2. Long version. Readers familiar with $[\mathrm{BDS}]$ and $[\mathrm{MP}]$ may skip to Section 3; others will find proofs of Theorems 1 and 2 in this section.

The set ${ }^{\omega} \omega$ of all mappings from $\omega$ to $\omega$ will be considered with the standard ordering, $f \leq^{*} g$ if $\{n \in \omega: f(n)>g(n)\}$ is finite. Recall that the bounding number $\mathfrak{b}$ is defined as $\min \left\{|F|: F \subseteq{ }^{\omega} \omega\right.$ is unbounded under $\left.\leq^{*}\right\}$; it is an uncountable regular cardinal and, given a mapping $g \in{ }^{\omega} \omega$ in advance, an unbounded family $\left\{f_{\alpha}: \alpha<\mathfrak{b}\right\}$ can be chosen so that (i) $f_{0} \geq g$, (ii) $f_{\alpha} \leq^{*} f_{\beta}$ and $f_{\beta} \mathbb{Z}^{*} f_{\alpha}$ for $\alpha<\beta<\mathfrak{b}$, and (iii) each $f_{\alpha}$ is nondecreasing.

Consider the set $\omega \times \omega$ with the partition $\mathcal{R}=\{\{n\} \times \omega: n \in \omega\}$. Fix an arbitrary $X \in \mathcal{J}^{+}(\mathcal{R})$. For $f \in{ }^{\omega} \omega$ define $[0, f)=\{\langle n, k\rangle \in \omega \times \omega: k<f(n)\}$. Choose $g \in{ }^{\omega} \omega$ so that $|X \cap[0, g)|=\omega$. Choose an unbounded family $\left\{f_{\alpha}: \alpha<\mathfrak{b}\right\}$ satisfying (i)-(iii) above. Now, the following hold:

(a) for every $\alpha<\mathfrak{b}$ and every $n \in \omega$, we have $\left|\left[0, f_{\alpha}\right) \cap(\{n\} \times \omega)\right|<\omega$;

(b) $\left|X \cap\left[0, f_{0}\right)\right|=\omega$;

(c) for every $P \in \mathcal{J}^{+}(\mathcal{R})$, the set

$$
H(P)=\left\{\alpha<\mathfrak{b}:(\forall \beta<\alpha)(\exists \gamma<\alpha)\left|P \cap\left[0, f_{\gamma}\right) \backslash\left[0, f_{\beta}\right)\right|=\omega\right\}
$$

is closed unbounded in $\mathfrak{b}$.

Indeed, (a) is trivial, (b) follows from (i) by our choice of the mapping $g$. For (c), consider any $P \in \mathcal{J}^{+}(\mathcal{R})$; we have to show that $H(P)$ is closed unbounded in $\mathfrak{b}$. Inasmuch as $\mathfrak{b}$ is an uncountable regular cardinal, it will suffice to show that, for each $\beta<\mathfrak{b}$, there exists $\gamma<\mathfrak{b}$ such that $\mid P \cap\left[0, f_{\gamma}\right) \backslash$ $\left[0, f_{\beta}\right) \mid=\omega$. Let $\beta<\mathfrak{b}$ be given. For each $n \in \omega$, choose $\left\langle m_{n}, k_{n}\right\rangle \in P \backslash\left[0, f_{\beta}\right)$ with $m_{n} \geq n$, and define $h(n)=k_{n}$. Choose $\gamma<\mathfrak{b}$ with $f_{\gamma} \mathbb{Z}^{*} h$. For any $n$ such that $f_{\gamma}(n)>h(n)$, we have $m_{n} \geq n$ and $f_{\gamma}\left(m_{n}\right) \geq f_{\gamma}(n)>h(n)=k_{n}$, and so $\left\langle m_{n}, k_{n}\right\rangle \in P \cap\left[0, f_{\gamma}\right) \backslash\left[0, f_{\beta}\right)$. Since $\left\{n: f_{\gamma}(n)>h(n)\right\}$ is infinite, it follows that $P \cap\left[0, f_{\gamma}\right) \backslash\left[0, f_{\beta}\right)$ is infinite.

Given a filter $\mathcal{F}$ on $\omega$, a set $X \subseteq \omega$ will be called $\mathcal{F}$-fat if, for each $F \in \mathcal{F}$, there exists $F^{\prime} \in \mathcal{F}$ with $\left|X \cap F \backslash F^{\prime}\right|=\omega$.

Notice that given a countably generated filter $\mathcal{F}$ other than a cofinite one, there are infinite sets $M$ satisfying $|M \backslash F|<\omega$ for every $F \in \mathcal{F}$ as well as infinite sets $N$ with $|N \cap F|<\omega$ for some $F \in \mathcal{F}$. A set $X$ is $\mathcal{F}$-fat if it contains both types of infinite sets as subsets.

Lemma 1. Let $\mathcal{F}$ be a countably generated free filter on $\omega$, and let $X$ be an $\mathcal{F}$-fat subset of $\omega$. Then there is a family $\left\{R_{\alpha}: \alpha<\mathfrak{b}\right\}$ of infinite subsets of $\omega$ such that:

(a) for each $\alpha<\mathfrak{b}$ and each $F \in \mathcal{F}$, we have $\left|R_{\alpha} \backslash F\right|<\omega$;

(a1) whenever $\alpha<\beta<\mathfrak{b}$, we have $\left|R_{\alpha} \backslash R_{\beta}\right|<\omega=\left|R_{\beta} \backslash R_{\alpha}\right|$;

(b) $R_{0} \subseteq X$; 
(c) for every $\mathcal{F}$-fat set $P \subseteq \omega$, the set

$$
\left\{\alpha<\mathfrak{b}:(\forall \beta<\alpha)(\exists \gamma<\alpha)\left|P \cap R_{\gamma} \backslash R_{\beta}\right|=\omega\right\}
$$

is closed unbounded in $\mathfrak{b}$.

Proof. As $X$ is $\mathcal{F}$-fat, the filter $F$ has a decreasing base $\left\{F_{n}: n \in \omega\right\}$ with $\left|X \cap F_{n} \backslash F_{n+1}\right|=\omega$ for each $n \in \omega$, and $F_{0}=\omega$. Identify $F_{n} \backslash F_{n+1}$ with $\{n\} \times \omega$, and follow the reasoning preceding Lemma 1. For $\alpha<\mathfrak{b}$ let $R_{\alpha}$ be the set corresponding to $\left[0, f_{\alpha}\right)$. Now (a), (a1), and (c) are clear, but (b) need not hold; we know only that $\left|X \cap R_{0}\right|=\omega$. To rectify this, replace $R_{0}$ with $X \cap R_{0}$.

Proof of Theorem 1. Let $T \subseteq<\omega_{1} \mathfrak{b}$ consist of the functions $s$ such that all values $s(\xi)$ are $\omega$-limits (limit ordinals of countable cofinality). Clearly, $T$ is a tree of height $\omega_{1}$ when ordered by $\subseteq$.

Our aim is to attach to each $s \in T$ a countably generated free filter $\mathcal{F}_{s}$ on $\omega$, an $\mathcal{F}_{s}$-fat set $X_{s} \subseteq \omega$, and an infinite set $A_{s} \subseteq \omega$; moreover, we will have $\mathcal{F}_{t} \subseteq \mathcal{F}_{s}$ whenever $t \subseteq s$, and $\left|A_{s} \backslash F\right|<\omega$ for all $F \in \mathcal{F}_{s}$. This will be done by a transfinite recursion of length $\omega_{1}$. For $\xi<\omega_{1}$, let $T_{\xi}=\{s \in T: \operatorname{dom} s=\xi\}$. For $s \in T_{\xi}$, the filter $\mathcal{F}_{s}$ and the set $X_{s}$ will be defined at step $\xi$, the set $A_{s}$ at step $\xi+1$.

STEP 0. Let $\mathcal{F}_{\emptyset}$ be any countably generated free filter on $\omega$ such that $\omega$ is $\mathcal{F}_{\emptyset}$-fat, and let $X_{\emptyset}=\omega$.

STEP $\eta+1$. We suppose that $\mathcal{F}_{s}$ and $X_{s}$ are known for all $s \in T_{\eta}$. For each $s \in T_{\eta}$, apply Lemma 1 to $\mathcal{F}_{s}$ and $X_{s}$, and let $\left\{R_{\alpha}^{s}: \alpha<\mathfrak{b}\right\}$ be the result. Let $A_{s}=R_{0}^{s}$; by Lemma 1 (a) we have $\left|A_{s} \backslash F\right|<\omega$ for all $F \in \mathcal{F}_{s}$. For each $\omega$-limit $\alpha<\mathfrak{b}$, let $\mathcal{F}_{s^{\urcorner} \alpha}$ be the smallest free filter on $\omega$ containing $\left\{R_{\alpha}^{s} \backslash R_{\beta}^{s}: \beta<\alpha\right\}$. Then $\mathcal{F}_{s^{\wedge} \alpha}$ is a countably generated filter extending $\mathcal{F}_{s}$, and $\omega$ is $\mathcal{F}_{s^{\wedge} \alpha}$-fat. Let $X_{s^{\wedge} \alpha}=\omega$.

STEP $\lambda$ a limit ordinal. We suppose that $\mathcal{F}_{t}$ is known for all $t \in \bigcup\left\{T_{\xi}\right.$ : $\xi<\lambda\}$. For each $s \in T_{\lambda}$, let $\mathcal{F}_{s}=\bigcup\left\{\mathcal{F}_{s \mid \xi}: \xi<\lambda\right\}$. Then $\mathcal{F}_{s}$ is a countably generated free filter on $\omega$, and $\omega$ is $\mathcal{F}_{s}$-fat. Define

$$
\mathcal{M}_{\lambda}=\left\{X \subseteq \omega: \mid\left\{s \in T_{\lambda}: X \text { is } \mathcal{F}_{s} \text {-fat }\right\} \mid=\mathfrak{c}\right\} .
$$

Assign to each $s \in T_{\lambda}$ an $\mathcal{F}_{s}$-fat set $X_{s} \subseteq \omega$ so that, for each $X \in \mathcal{M}_{\lambda}$, we have $X=X_{s}$ for some $s \in T_{\lambda}$.

Let $\mathcal{A}=\left\{A_{s}: s \in T\right\}$. Then $\mathcal{A}$ is infinite, in fact, $|\mathcal{A}|=|T|=\mathfrak{c}$. We will show that $\mathcal{A}$ is almost disjoint and completely separable. For $s, t \in T, s \neq t$, let $\xi(s, t)$ be the maximal ordinal $\xi$ with $s\lceil\xi=t\lceil\xi$.

$\mathcal{A}$ is almost disjoint: Suppose $u, v \in T, u \neq v$. Let $\xi=\xi(u, v)$ and $s=$ $u|\xi=v| \xi$. We may assume that either $v=s$ and $u$ is a proper extension of $s$, or else both $u$ and $v$ are proper extensions of $s$ and $v(\xi)<u(\xi)$. Let $\alpha=u(\xi)$; 
let $\beta=0$ if $v=s$, and $\beta=v(\xi)$ otherwise. Then $R_{\alpha}^{s} \backslash R_{\beta}^{s} \in \mathcal{F}_{s^{\wedge} \alpha} \subseteq \mathcal{F}_{u}$ and, if $\beta>0, R_{\beta}^{s} \in \mathcal{F}_{s^{\wedge} \beta} \subseteq \mathcal{F}_{v}$. Thus $\left|A_{u} \backslash\left(R_{\alpha}^{s} \backslash R_{\beta}^{s}\right)\right|<\omega$ and $\left|A_{v} \backslash R_{\beta}^{s}\right|<\omega$, whence $\left|A_{u} \cap A_{v}\right|<\omega$.

If $X \in \mathcal{J}^{+}(\mathcal{A})$, then there is some $t \in T$ such that $X$ is $\mathcal{F}_{t}$-fat: Let $S=\left\{s \in T:\left|X \cap A_{s}\right|=\omega\right\}$. It is easy to see (e.g., using Ramsey's theorem) that there is an infinite set $\left\{s_{i}: i \in \omega\right\}$ of distinct elements of $S$ such that either (1) for some ordinal $\xi$ we have $\xi\left(s_{i}, s_{j}\right)=\xi$ whenever $i<j$, or else (2) for some ordinals $\xi_{i}, i \in \omega$, we have $\xi\left(s_{i}, s_{j}\right)=\xi_{i}<\xi_{j}$ whenever $i<j$.

Suppose (1) holds. Then there is some $s \in T_{\xi}$ such that $s_{i} \mid \xi=s$ for all $i \in \omega$. We may assume that each $s_{i}$ is a proper extension of $s$; let $\beta_{i}=s_{i}(\xi)$. We may assume that $\beta_{i}<\beta_{j}$ whenever $i<j$. Let $\alpha=\sup \left\{\beta_{i}: i \in \omega\right\}$ and $t=s^{\curvearrowleft} \alpha$; we claim that $X$ is $\mathcal{F}_{t}$-fat.

Consider any $F \in \mathcal{F}_{t}$. Then $F \supseteq\left(R_{\alpha}^{s} \backslash R_{\beta_{i}}^{s}\right) \backslash D$ for some $i \in \omega$ and some finite set $D$. Let $j=i+1$ and let $F^{\prime}=R_{\alpha}^{s} \backslash R_{\beta_{j}}^{s} \in \mathcal{F}_{t}$. Then $R_{\beta_{j}}^{s} \backslash R_{\beta_{i}}^{s} \in$ $\mathcal{F}_{s^{\wedge} \beta_{j}} \subseteq \mathcal{F}_{s_{j}}$, and so $\left|A_{s_{j}} \backslash\left(R_{\beta_{j}}^{s} \backslash R_{\beta_{i}}^{s}\right)\right|<\omega$. Since $A_{s_{j}} \backslash\left(F \backslash F^{\prime}\right) \subseteq\left[A_{s_{j}} \backslash\right.$ $\left.\left(R_{\beta_{j}}^{s} \backslash R_{\beta_{i}}^{s}\right)\right] \cup\left(R_{\beta_{j}}^{s} \backslash R_{\alpha}^{s}\right) \cup D$, it follows that $\left|A_{s_{j}} \backslash\left(F \backslash F^{\prime}\right)\right|<\omega$. Since $\left|X \cap A_{s_{j}}\right|=\omega$, we also have $\left|X \cap F \backslash F^{\prime}\right|=\omega$, as required.

Now suppose (2) holds. For $i \in \omega$ let $t_{i}=s_{i} \mid \xi_{i}$, and let $t=\bigcup\left\{t_{i}: i \in \omega\right\}$, so that $\mathcal{F}_{t}=\bigcup\left\{\mathcal{F}_{t_{i}}: i \in \omega\right\}$. We claim that $X$ is $\mathcal{F}_{t}$-fat.

Consider any $F \in \mathcal{F}_{t}$. For some $i \in \omega$ we have $F \in \mathcal{F}_{t_{i}} \subseteq \mathcal{F}_{s_{i}}$, and so $\left|A_{s_{i}} \backslash F\right|<\omega$. Let $\alpha=t\left(\xi_{i}\right)$. We consider three cases:

If $s_{i}=t_{i}$, then $A_{s_{i}}=R_{0}^{t_{i}}$; in this case let $F^{\prime}=R_{\alpha}^{t_{i}} \backslash R_{0}^{t_{i}}$.

If $s_{i} \supseteq t_{i}^{\Upsilon} \beta$ where $\beta<\alpha$, then $R_{\beta}^{t_{i}} \in \mathcal{F}_{t_{i} \beta} \subseteq \mathcal{F}_{s_{i}}$ and so $\left|A_{s_{i}} \backslash R_{\beta}^{t_{i}}\right|<\omega$; in this case let $F^{\prime}=R_{\alpha}^{t_{i}} \backslash R_{\beta}^{t_{i}}$.

If $s_{i} \supseteq t_{i}^{\frown \beta}$ where $\beta>\alpha$, then $R_{\beta}^{t_{i}} \backslash R_{\alpha}^{t_{i}} \in \mathcal{F}_{t_{i}^{\wedge} \beta} \subseteq \mathcal{F}_{s_{i}}$ and so $\mid A_{s_{i}} \backslash$ $\left(R_{\beta}^{t_{i}} \backslash R_{\alpha}^{t_{i}}\right) \mid<\omega$; in this case let $F^{\prime}=R_{\alpha}^{t_{i}}$.

In every case we have $F^{\prime} \in \mathcal{F}_{t_{i}^{\prime} \alpha} \subseteq \mathcal{F}_{t}$ and $\left|A_{s_{i}} \cap F^{\prime}\right|<\omega$, and so $\left|A_{s_{i}} \backslash\left(F \backslash F^{\prime}\right)\right|<\omega$. Since $\left|X \cap A_{s_{i}}\right|=\omega$, it follows that $\left|X \cap F \backslash F^{\prime}\right|=\omega$, as required.

$\mathcal{A}$ is completely separable: We have just shown that, if $X \in \mathcal{J}^{+}(\mathcal{A})$, then there exist $\xi<\omega_{1}$ and $s_{\emptyset} \in T_{\xi}$ such that $X$ is $\mathcal{F}_{s_{\emptyset}}$-fat. By the construction

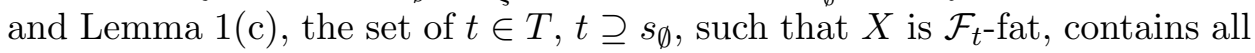
extensions $s_{\emptyset} \propto \alpha$ with $\alpha$ ranging in an unbounded subset of $\mathfrak{b}$. In particular, there are two different extensions $s_{0}, s_{1}$ of $s_{\emptyset}$ to $\xi+1$ such that $X$ is both $\mathcal{F}_{s_{0}}$-fat and $\mathcal{F}_{s_{1}}$-fat. This is the start of a branching process which ends at level $\lambda=\xi+\omega$, where $X \in \mathcal{M}_{\lambda}$. Now, by the construction, we have $X=X_{s}$ for some $s \in T_{\lambda}$, and so $A_{s}=R_{0}^{s} \subseteq X_{s}=X$.

Proof of Theorem 2. Let $\mathcal{A}_{0}=\{\omega\}$. If the family $\mathcal{A}_{n} \subseteq[\omega]^{\omega}$ is known, choose for each $A \in \mathcal{A}_{n}$ an infinite completely separable almost disjoint 
family $\mathcal{A}_{A}$ on $A$ and let $\mathcal{A}_{n+1}=\bigcup\left\{\mathcal{A}_{A}: A \in \mathcal{A}_{n}\right\}$. This defines a sequence $\left\{\mathcal{A}_{n}: n \in \omega\right\}$ of almost disjoint families on $\omega$.

For $n>0$, fix a one-to-one mapping $f_{n}: \mathcal{J}^{+}\left(\mathcal{A}_{n}\right) \cap \bigcup\left\{\mathcal{P}(A): A \in \mathcal{A}_{n-1}\right\}$ $\rightarrow \mathcal{A}_{n}$ such that $f_{n}(X) \subsetneq X$ for each $X \in \operatorname{dom} f_{n}$. (By our choice of the families $\mathcal{A}_{n}$, this is possible according to Observation 1.) Define a mapping $\psi: \mathcal{P}(\omega) \rightarrow \mathcal{P}(\omega)$ by the formula

$$
\psi(P)=\bigcup_{n \in \omega} \bigcup\left\{X \in \operatorname{dom} f_{n}: P \cap f_{n}(X) \in \mathcal{J}^{+}\left(\mathcal{A}_{n+1}\right)\right\} ;
$$

thus $\psi\left(f_{n}(X)\right) \supseteq X$ whenever $X \in \operatorname{dom} f_{n}$. (In fact $\psi\left(f_{n}(X)\right)=X$, as the interested reader can easily verify.) Clearly $\psi(\emptyset)=\emptyset$, and $\psi(P \cup Q)=$ $\psi(P) \cup \psi(Q)$ for all $P, Q \subseteq \omega$. Hence, the mapping $\varphi(P)=P \cup \psi(P)$ is a closure function; we claim that $\varphi$ is a Cech function. The mapping $\varphi$ is not the identity, since $\omega=\psi\left(f_{1}(\omega)\right)=\varphi\left(f_{1}(\omega)\right)$ and $f_{1}(\omega) \neq \omega$.

The mapping $\varphi$ maps $\mathcal{P}(\omega)$ onto $\mathcal{P}(\omega)$ : Let $Y \subseteq \omega$. For $n \in \omega$, let $\mathcal{B}_{n}=\left\{A \in \mathcal{A}_{n}: Y \cap A \in \mathcal{J}^{+}\left(\mathcal{A}_{n+1}\right)\right\}$. Let $\mathcal{B}=\bigcup\left\{\mathcal{B}_{n}: n \in \omega\right\}$, and let $\mathcal{C}$ be the set of all maximal elements of $\mathcal{B}$. For $C \in \mathcal{C}$, let $n(C)$ be the unique $n \in \omega$ with $C \in \mathcal{A}_{n}$.

For each $n \in \omega$, the set $\left\{A \in \mathcal{A}_{n}:(\exists C \in \mathcal{C}) C \subseteq A\right\}$ is finite: This is clear for $n=0$, since $\left|\mathcal{A}_{0}\right|=1$. Suppose that $\left\{A \in \mathcal{A}_{n}:(\exists C \in \mathcal{C}) C \subseteq A\right\}=$ $\left\{A_{i}: i<k\right\}$, while $\mathcal{D}=\left\{D \in \mathcal{A}_{n+1}:(\exists C \in \mathcal{C}) C \subseteq D\right\}$ is infinite. Then $\left\{D \in \mathcal{D}: D \subseteq A_{i}\right\}$ is infinite for some $i<k$. But then $A_{i} \in \mathcal{B}_{n}$, and we have some $C^{\prime} \in \mathcal{C}$ with $C^{\prime} \supseteq A_{i}$ as well as some $C \in \mathcal{C}$ with $C \subsetneq A_{i}$, which is impossible. Induction completes the proof.

For $C \in \mathcal{C} \cap \mathcal{A}_{n}$, let $Y(C)=Y \cap C \backslash \bigcup\{E \in \mathcal{C}: n(E)<n\}$. Then $|(Y \cap C) \backslash Y(C)|<\omega$, since $\mathcal{C}$ is almost disjoint and for each $k \in \omega$ contains only finitely many elements of $\mathcal{A}_{k}$; hence $Y(C) \in \operatorname{dom} f_{n+1}$. Let

$$
Q=\bigcup\left\{f_{n+1}(Y(C)): n \in \omega, C \in \mathcal{C} \cap \mathcal{A}_{n}\right\} \subseteq Y,
$$

and let $R=Y \backslash \psi(Q)$. We claim that $Y=\varphi(Q \cup R)$. As $\varphi(Q \cup R)=$ $Q \cup R \cup \psi(Q \cup R)=Q \cup(Y \backslash \psi(Q)) \cup \psi(Q) \cup \psi(R)=Q \cup Y \cup \psi(Q) \cup \psi(R)=$ $Y \cup \psi(Q) \cup \psi(R)$, we will finish the proof by showing that $\psi(R)=\emptyset$ and $\psi(Q) \subseteq Y$.

$\psi(R)=\emptyset$ : Assuming $\psi(R) \neq \emptyset$, we must have $R \cap f_{n}(X) \in \mathcal{J}^{+}\left(\mathcal{A}_{n+1}\right)$ for some $n \in \omega$ and $X \in \operatorname{dom} f_{n}$. Since $R \subseteq Y$, it follows that $f_{n}(X) \in \mathcal{B}_{n}$, and so $f_{n}(X) \subseteq C$ for some $C \in \mathcal{C}$; let $m=n(C)$. Then $f_{m+1}(Y(C)) \subseteq Q$, and so we have $Y(C) \subseteq \psi\left(f_{m+1}(Y(C))\right) \subseteq \psi(Q)$ and $R \cap f_{n}(X)=Y \cap$ $f_{n}(X) \backslash \psi(Q) \subseteq Y \cap C \backslash Y(C)$. Hence $\left|R \cap f_{n}(X)\right| \leq|Y \cap C \backslash Y(C)|<\omega$, contradicting the assumption that $R \cap f_{n}(X) \in \mathcal{J}^{+}\left(\mathcal{A}_{n+1}\right)$.

$\psi(Q) \subseteq Y$ : It will suffice to show that, if $X \in \operatorname{dom} f_{n}$ and $Q \cap f_{n}(X) \in$ $\mathcal{J}^{+}\left(\mathcal{A}_{n+1}\right)$, then $X \subseteq Y$. Consider such an $X$, and let $B=f_{n}(X) \in \mathcal{A}_{n}$. 
Since $Q \subseteq Y$, it follows that $B \in \mathcal{B}_{n}$, and so $B \subseteq C$ for some $C \in \mathcal{C}$; let $m=n(C) \leq n$.

If $E \in \mathcal{C} \backslash\{C\}$, then $Y(E) \cap C$ is finite since $E \cap C$ is finite; moreover, $Y(E) \cap C=\emptyset$ except for finitely many sets $E$ with $n(E) \leq m$. Hence the set $\bigcup\{Y(E): E \in \mathcal{C} \backslash\{C\}\}$ has finite intersection with $C$, and therefore also with $B$. Since $B \cap Q \in \mathcal{J}^{+}\left(\mathcal{A}_{n+1}\right)$, we must have $B \cap f_{m+1}(Y(C)) \in$ $\mathcal{J}^{+}\left(\mathcal{A}_{n+1}\right)$.

Let $D=f_{m+1}(Y(C)) \in \mathcal{A}_{m+1}$. We consider three cases:

If $m=n$, then $D \in \mathcal{A}_{n+1}$; this is impossible since $B \cap D \in \mathcal{J}^{+}\left(\mathcal{A}_{n+1}\right)$.

If $m=n-1$, then $D \in \mathcal{A}_{n}$. Since $B \cap D$ is infinite, we must have $B=D$, i.e., $f_{n}(X)=f_{n}(Y(C))$. Since $f_{n}$ is one-to-one, it follows that $X=Y(C) \subseteq Y$.

If $m<n-1$, then $D \in \mathcal{A}_{k}$ where $k=m+1 \leq n-1$. Since $X \in \operatorname{dom} f_{n}$, we have $X \subseteq A$ for some $A \in \mathcal{A}_{n-1}$. Since $A \cap D$ is infinite, we must have $A \subseteq D$, and so $X \subseteq D \subseteq Y(C) \subseteq Y$.

3. Concluding remarks. (a) It should be obvious that, once we have a Cech function on $\omega$, then we also have a Čech function $\varphi$ on any $\kappa \geq \omega$, and we may even demand that $|\varphi(P) \backslash P|=\kappa$ for some $P \subseteq \kappa$. The situation changes if we demand that $\varphi$ be $\kappa$-additive, i.e., whenever $\tau<\kappa$, then $\varphi\left(\bigcup_{\iota<\tau} P_{\iota}\right)=$ $\bigcup_{\iota<\tau} \varphi\left(P_{\iota}\right)$. The foregoing construction generalizes to a regular cardinal $\kappa$ under the assumption that there is a completely separable almost disjoint family $\mathcal{A}$ on $\kappa$; this means that $|A \cap B|<\kappa$ for distinct $A, B \in \mathcal{A}$, every $A \in \mathcal{A}$ is of size $\kappa$, and every $X \subseteq \kappa$ such that $|\{A \in \mathcal{A}:|X \cap A|=\kappa\}| \geq \kappa$ satisfies $|\{A \in \mathcal{A}: A \subseteq X\}|=2^{\kappa}$. But there is no hope of finding such a family in ZFC, since James E. Baumgartner [B] has shown that " $2^{\omega_{1}}>\omega_{2}$ and there is no almost disjoint family on $\omega_{1}$ of size $\omega_{3}$ " is consistent with ZFC. In the case of a measurable cardinal $\kappa$, Roderick A. Price [P, Theorem 3.13] has shown that a $\kappa$-additive Čech function $\varphi: \mathcal{P}(\kappa) \rightarrow \mathcal{P}(\kappa)$ exists if $\kappa$ carries a $\kappa$-complete ultrafilter of character $\kappa^{+}$.

(b) The reader may have noticed that our Čech function has plenty of fixed points. This is not some strange by-product of the construction. Jiří Vinárek proved in the seventies the following unpublished statement:

Theorem (Vinárek). If $\varphi$ is a Čech function on $X$, then for every infinite $P \subseteq X$ there is an infinite $T \subseteq P$ such that $\varphi(S)=S$ for all $S \subseteq T$. (That is, every infinite subset of $X$ contains an infinite discrete closed set.)

Proof. Choose a countably infinite set $P_{0}=\left\{x_{n}: n \in \omega\right\} \subseteq P$. For each $n \in \omega$ choose $P_{n+1}$ with $\varphi\left(P_{n+1}\right)=P_{n} \backslash\left\{x_{n}\right\}$. Thus $\left\{P_{n}: n \in \omega\right\}$ is a decreasing sequence of infinite sets, and $\bigcap\left\{\varphi\left(P_{n}\right): n \in \omega\right\}=\emptyset$. Choose $t_{n} \in P_{n} \backslash\left\{t_{0}, \ldots, t_{n-1}\right\}$ and let $T=\left\{t_{n}: n \in \omega\right\}$; thus $\left|T \backslash P_{n}\right| \leq n$ for all $n$. If $S \subseteq T$, then $\varphi(S) \subseteq S \cup \varphi\left(P_{n}\right)$ for every $n$, whence $\varphi(S)=S$. 
Note that Vinárek's theorem holds true not only for a Čech function, but also for any closure function $\varphi$ satisfying $(1) \varphi(\{x\})=\{x\}$ for all $x \in X$, and (2) for each infinite set $P \subseteq X$ there is an infinite set $Q$ with $\varphi(Q) \subseteq P$.

(c) Although our proof makes heavy use of the axiom of choice, we do not know if the existence of a Čech function on $\omega$ is in fact independent of ZF.

\section{References}

[BDS] B. Balcar, J. Dočkálková, and P. Simon, Almost disjoint families of countable sets, in: Finite and Infinite Sets (Eger, 1981), Colloq. Math. Soc. János Bolyai 37, North-Holland, Amsterdam, 1984, 59-88.

[B] J. E. Baumgartner, Almost-disjoint sets, the dense set problem and the partition calculus, Ann. Math. Logic 9 (1976), 401-439.

[Č $\quad$ E. Čech, Problème 75, Fund. Math. 34 (1947), 332.

[G] F. Galvin, On a problem of Čech, Notices Amer. Math. Soc. 25 (1978), A-604 and A-720.

$[\mathrm{H}] \mathrm{S} . \mathrm{H}$. Hechler, Classifying almost-disjoint families with applications to $\beta N-N$, Israel J. Math 10 (1971), 413-432.

[K] K. Kunen, Set Theory, North-Holland, Amsterdam, 1980.

[MP] E. C. Milner and K. Prikry, Almost disjoint sets, in: Surveys in Combinatorics 1987, London Math. Soc. Lecture Note Ser. 123, Cambridge Univ. Press, Cambridge, 1987, 155-172.

[P] R. A. Price, On a problem of Čech, Topology Appl. 14 (1982), 319-329.

Department of Mathematics

University of Kansas

1460 Jayhawk Blvd.

Lawrence, KS 66045-7523, U.S.A.

E-mail: galvin@math.ku.edu
Faculty of Mathematics and Physics

Charles University Malostranské nám. 25 11800 Praha 1, Czech Republic E-mail: psimon@ms.mff.cuni.cz

Received 24 August 2005;

in revised form 5 September, 2006 